\title{
DIFFERENTES SOURCES D'ERREURS DANS LE DIAGNOSTIC DE PERFORMANCE ÉNERGÉTIQUE POUR LES BÂTIMENTS
}

\author{
Author(s) / Auteur(s) : \\ Richard CANTIN \\ Ecole Nationale des Travaux Publics de l'Etat, Université de Lyon \\ Rue Maurice Audin 60120 Vaulx-en-Velin, France \\ richard.cantin@entpe.fr \\ Cédric BEREAUD \\ Ecole Nationale des Travaux Publics de l'Etat, Université de Lyon \\ Rue Maurice Audin 60120 Vaulx-en-Velin, France \\ cedric.bereaud@entpe.fr
}

\section{Résumé :}

Parmi les différents diagnostics mis en oeuvre dans le bâtiment, le diagnostic de performance énergétique (DPE) renseigne sur la performance énergétique en évaluant sa consommation d'énergie et son impact en termes d'émission de gaz à effet de serre.

Le contenu et les modalités d'établissement du DPE sont réglementés en France depuis plus de 10 ans. Les DPE ont ainsi produit des données, des rapports, des guides, des logiciels... utilisés pour élaborer les stratégies de réhabilitation énergétique du parc immobilier.

Mais aujourd'hui les retours d'expériences interrogent la qualité de ces DPE. En effet, alors que ces diagnostics influencent le marché de l'immobilier, différentes enquêtes ont montré qu'ils manquaient de fiabilité... Dans ces conditions, il semble difficile d'assurer la qualité des prises de décision pour une transition énergétique efficace dans le secteur du bâtiment.

Il apparaît que l'évaluation de la performance énergétique des bâtiments relève d'une problématique complexe qui fait appel à un ensemble de connaissances dans des domaines variés. Définir la performance énergétique d'un bâtiment renvoie à toutes les difficultés de compréhension posées par l'appréhension d'une réalité complexe.

Cet article présente comment la vision systémique permet d'appréhender la problématique complexe de l'évaluation de la performance énergétique des bâtiments. Avec plusieurs exemples, il montre comment la modélisation des systèmes, l'identification des facteurs complexes et leurs influences vont permettre de caractériser les différentes sources d'erreurs. Enfin, l'article propose des actions visant à réduire ces sources d'erreurs et à améliorer l'intégration des exigences du développement durable dans le secteur du bâtiment.

Mots-clés :

diagnostic, bâtiment, performance, énergie, erreur, systémique

\section{INTRODUCTION}

En France, afin de protéger et d'informer un futur propriétaire ou locataire sur les éléments d'un immeuble susceptible de présenter des risques pour la santé ou pour la sécurité des personnes, un dossier de diagnostic technique a été rendu obligatoire par le code de la construction et de l'habitat. Ce dossier comprend plusieurs diagnostics techniques immobiliers : état de l'installation intérieure d'électricité et de gaz, constat de risque d'exposition au plomb, état d'amiante, état de présence de termites, état de risques naturels et technologiques, état des installations d'assainissement non collectif et diagnostic de performance énergétique (DPE) (MTES, 2018a).

Depuis plus de 10 ans, le contenu et les modalités d'établissement du DPE sont réglementés. Il renseigne sur la performance énergétique d'un logement ou d'un bâtiment, en évaluant sa consommation d'énergie et son impact en termes d'émissions de gaz à effet de serre. La lecture du DPE est facilitée par une étiquette «Energie » et une étiquette «Climat » à sept classes de A à G. Ce 
diagnostic, établi par un professionnel indépendant satisfaisant à des critères de compétence, s'inscrit dans le cadre de la politique énergétique définie au niveau européen afin de réduire la consommation d'énergie des bâtiments et de limiter les émissions de gaz à effet de serre (MTES, 2018b).

Mais plusieurs erreurs dans les DPE ont été révélées par des enquêtes comme celles réalisées par l'association UFC-que Choisir en 2008, 2011, 2012 et 2017 (UFC, 2017). L'étude réalisée en 2017 sur sept maisons réparties sur le territoire français a révélé des divergences nombreuses au niveau :

- de la durée des visites sur site : la durée de la visite peut être très variable, le diagnostic étant parfois réalisé en une vingtaine de minutes alors qu'il y a une soixantaine de points de contrôle à vérifier ;

- de la classe énergétique du logement: aucune des 7 maisons n'obtient la même classe énergétique avec les différents diagnostiqueurs immobiliers certifiés ;

- de l'identification des sources de déperditions thermiques ;

- de la prise en compte des énergies renouvelables ;

- des recommandations d'amélioration énergétique.

D'autres études confirment le manque de fiabilité des diagnostics de performance énergétique des bâtiments. Par exemple, en Italie, un même bâtiment a été étudié par 162 diagnostiqueurs. Même si plus de $70 \%$ des experts ont classé le bâtiment avec la même étiquette énergétique $\mathrm{D}$, les résultats varient de la classe A à la classe E (Tronchin \& Fabbri, 2012).

Les impacts économiques des DPE ne sont pas négligeables. Par exemple, l'étiquette «Energie » a une influence sur le prix du logement. Une étude réalisée en 2013 sur un panel de plus de 300000 logements au Royaume-Uni met en évidence l'impact de l'étiquette énergétique sur le prix du logement (Fuerst \& all, 2013). Les résultats montrent que, comparé à un logement classé G, le logement classé $\mathrm{E}$ ou $\mathrm{F}$ se vend $6 \%$ plus cher; le logement classé $\mathrm{D}$ se vend $8 \%$ plus cher; le logement classé C se vend 10\% plus cher, et le logement classé B ou A se vend 14\% plus cher. En France, une étude similaire a montré un écart de prix de 5\% en moyenne par classe du DPE avec des écarts variables selon les régions (DINAMIC, 2015).

Ces différents résultats génèrent une méfiance des ménages quant à la qualité des diagnostics. Des études montrent une bonne connaissance des EPC (Energy Performance Certificate, équivalent du DPE dans d'autres pays européens), mais une confiance limitée en ces derniers. Par exemple, en Allemagne, une enquête réalisée en 2012 a montré que $80 \%$ des ménages connaissaient les EPC mais que moins de la moitié d'entre eux avaient confiance dans les résultats donnés (Amecke, 2012).

Alors que ces DPE produisent des données, des recommandations, des rapports, des guides, des logiciels et des statistiques... les études et les retours d'expériences interrogent la finalité et l'usage de ces représentations (Ministère du Logement, 2009., Cantin et al., 2013., Bjorn et al., 2016., Jenkins et al. 2017., ADEME, 2018). Dans ce contexte, il est difficile d'assurer la qualité des prises de décision pour réhabiliter des bâtiments. Se posent alors des questions relatives à la caractérisation des sources d'erreurs qui compromettent la fiabilité du diagnostic et l'efficacité des actions en faveur d'un développement durable des territoires.

La littérature scientifique traitant des problématiques relatives aux erreurs de diagnostics montre qu'elles concernent de nombreux domaines. Par exemple, le domaine de la médecine fournit de multiples études à ce sujet, les diagnostics médicaux étant sujets à une amélioration constante. En permanence, il faut identifier les causes d'un diagnostic erroné, et il est parfois extrêmement difficile de détecter les erreurs mais aussi d'en comprendre les causes tant elles sont complexes et multifactorielles (Tudela and al., 2017). Les erreurs dans les diagnostics sont souvent dues à une combinaison d'erreurs cognitives difficilement quantifiables (Thammasitboon and al., 2013). C'est pourquoi il convient d'identifier les facteurs susceptibles d'entraîner des erreurs de diagnostic même ceux qui semblent avoir un impact limité de prime abord. 


\section{RECENSEMENT DES VARIABLES DU SYSTEME DPE}

\section{Démarche adoptée}

Les retours d'expériences montrent que les sources d'erreurs dans le DPE pour les bâtiments sont multifactorielles. L'évaluation de la performance énergétique des bâtiments relève d'une problématique complexe qui fait appel à un ensemble de connaissances dans des domaines variés. Définir la performance énergétique d'un bâtiment renvoie à toutes les difficultés de compréhension et de modélisation d'une réalité complexe.

Dans ce contexte, pour considérer et affronter la complexité, l'approche systémique fournit des concepts et des outils (Rosnay, 1975., Morin, 1977., Durand, 1994., Le Moigne 1994 et 1995., Donnadieu et al., 2003). Elle peut proposer une nouvelle vision du DPE, l'exercice de la pensée complexe aidant à l'intelligence des systèmes complexes (Perriault, J. et al., 2011).

Parmi les outils de la systémique, l'analyse structurelle offre, à l'aide de la méthode dite MICMAC «Matrice d'Impacts Croisés Multiplication Appliquée à un Classement (Godet, 1991), la possibilité de décrire un système avec une matrice mettant en relation les éléments constitutifs de ce système. Ainsi le système DPE est défini par des variables et par les relations qui existent entre elles.

Partant de cette description, cette démarche vise à faire apparaître les principales variables influentes et dépendantes puis les variables essentielles à l'évolution du système, chaque variable étant en relation avec les autres variables. L'intérêt de la méthode repose sur l'idée que l'influence d'une variable sur le système tient plus des différentes relations indirectes que la variable peut avoir avec les autres variables que de son influence directe. Michel Godet le résume en disant «Dans une vision systémique du monde, une variable n'existe que par rapport à ses relations » (Godet, 1991).

\section{Liste des variables et des influences}

Pour appréhender les différentes sources d'erreurs influençant la qualité du DPE, une liste de 18 variables a été établie à partir des retours d'expériences et de questions soulevées quant à la qualité du DPE.

La Table 1 présente les variables associées aux sources d'erreurs, le questionnement associé à chacune d'elle, et leurs influences directes sur d'autres variables. Ces influences directes des variables sur les autres sont brièvement commentées. Par exemple, la variable «1. Année de construction » influence directement les variables «3. méthode de calcul utilisée », «9. Durée du diagnostic », «7. Qualité et pércision des données » et «11. Visite sur site ».

\begin{tabular}{|c|c|c|}
\hline \multirow[b]{2}{*}{$\mathbf{N}^{\circ}$} & \multicolumn{2}{|c|}{ Questionnement } \\
\hline & $\begin{array}{l}\text { Variable } \\
\text { (Abréviation) }\end{array}$ & $\begin{array}{l}\text { Commentaires sur les influences directes de la variable sur } \\
\text { - les autres variables... }\end{array}$ \\
\hline \multirow[b]{2}{*}{1} & \multicolumn{2}{|c|}{ En quelle année a été construit le bâtiment? } \\
\hline & $\begin{array}{l}\text { Année de } \\
\text { construction } \\
\text { (Annee) }\end{array}$ & $\begin{array}{l}\text { - Méthode de calcul utilisée : la réglementation impose l'utilisation d'une méthode en fonction de l'année } \\
\text { de construction lorsque l'année est saisie } \\
\text { - Durée du diagnostic : les bâtiments plus anciens demandent plus de temps pour mener le diagnostic du } \\
\text { fait de systèmes constructifs complexes. } \\
\text { - Qualité et précision des données : pour un bâtiment récent, les données devraient être a priori facilement } \\
\text { disponibles et plus précises } \\
\text { - Visite sur site : plusieurs retours d'expériences montrent l'absence de visite sur site notamment pour les } \\
\text { bâtiments anciens }\end{array}$ \\
\hline & \multicolumn{2}{|c|}{ Quel logiciel est utilisé ? } \\
\hline 2 & $\begin{array}{l}\text { Logiciel utilisé } \\
\text { (Logiciel) }\end{array}$ & $\begin{array}{l}\text { - Nombre de données renseignées : l'interface du logiciel ainsi que la logique de saisie des données a une } \\
\text { influence sur le nombre de données renseignées par le diagnostiqueur, notamment le nombre de champs } \\
\text { renseigné «inconnu » } \\
\text { - Durée du diagnostic : la prise en main du logiciel ainsi que son interface conduisent à un diagnostic plus } \\
\text { ou moins rapide }\end{array}$ \\
\hline
\end{tabular}


Quelle méthode de calcul est utilisée?

3 Méthode de calcul

Nombre de données renseignées : la méthode des factures ne requiert que très peu de données alors que la méthode conventionnelle demande un travail plus important

- Qualité et précisions des données : la méthode des factures utilise des données réelles de consommation

(Metho cal) alors que la méthode conventionnelle repose sur des paramètres renseignés par le diagnostiqueur, sur la base de documents, de mesures et sur les dires du propriétaire

- Durée du diagnostic : la méthode des factures est plus rapide que la méthode calculatoire conventionnelle

Les compétences du diagnostiqueur suffisent-elles pour réaliser le diagnostic?

- - Qualité et précisions des données : un diagnostiqueur avec un niveau élevé de connaissances aura une compréhension plus avancée de la physique énergétique complexe du bâtiment

- Nombre de données renseignées : un diagnostiqueur qualifié saisira a priori moins de champs de données comme « inconnues »

4 Qualification du

Logiciel utilisé : les logiciels simples et intuitifs existent, d'autres sont plus avancés et permettent une diagnostiqueur saisie précise. Les compétences et les objectifs du diagnostiqueur auront une incidence sur le choix du logiciel

(Qualif)

- Méthode de calcul utilisée : l'utilisation d'une méthode est réglementée mais différents retours d'expériences montrent que ce n'est pas toujours respecté

- Durée du diagnostic : les compétences du diagnostiqueur auront une incidence sur sa capacité à réaliser le diagnostic plus ou moins rapidement

- Visite sur site : elle est obligatoire. Les compétences et les objectifs du diagnostiqueur joueront un rôle dans le respect de cette obligation

Les documents disponibles sont-ils suffisants ? Répondent-ils aux attentes des diagnostiqueurs ?

5

Documents à

disposition des

diagnostiqueurs

(Doc dispo)

- Qualité et précision des données : La qualité des données renseignées dépend de l'adéquation entre les documents disponibles et la réalité du terrain

- Nombre de données renseignées : les documents et guides devraient aider le diagnostiqueur à lever les doutes et éviter la saisie de données dites « inconnues » lors de son diagnostic

- Visite sur site : les documents devraient aider le diagnostiqueur à réaliser sa visite sur site de manière à ce qu'il puisse contrôler les points nécessaires à la réalisation du DPE

Combien de données sont renseignées ? Combien sont renseignées comme "inconnues" ?

6

Nombre de

données

renseignées

(Données re)

- Base de données : leur fiabilité est fortement influencée par le contenu du DPE, c'est-à-dire par les données saisies

Quelle est la provenance des données ? Combien de données sont renseignées "au jugé" ?

7

Qualité et

précision des

données

Base de données : elle est influencée par la qualité et la précision des différentes données saisies (Stone

(Precis don)

Les bases de données sont-elles fiables ?

8 Base de données

Objectifs énergétiques : L'évaluation des avancées vers des objectifs de réduction des consommation

(Bases don) - Politiques publiques : les résultats issus des bases de données influencent la définition et la mise en œuvre des actions, des plans et des politiques relatives à la transition énergétique : actions à mener, résultats considérés comme « bons », « encourageants », « mauvais », etc.

Quelle est la durée du diagnostic ?

9 Durée du diagnostic (Duree dia)

Nombre de données renseignées : avec une soixantaine de points de contrôle, le nombre de données renseignées sera influencé par la durée du diagnostic

- Qualité et précisions des données : la recherche de la qualité et de la précision des données demande plus d'attention, d'effort et de temps

Est-ce un DPE obligatoire, volontaire, pour la vente, pour la location ?

10 Objectifs du DPE Qualité et précisions des données : les retours d'expérience montrent qu'un DPE effectué sans contrôle peut parfois être réalisé avec moins de minuties

(Objectifs)

- Durée du diagnostic : un DPE incontrôlé est parfois réalisé rapidement

- Objectifs environnementaux : un DPE réalisé volontairement sans obligation révèle une motivation à maitriser la consommation énergétique et les impacts environnementaux 


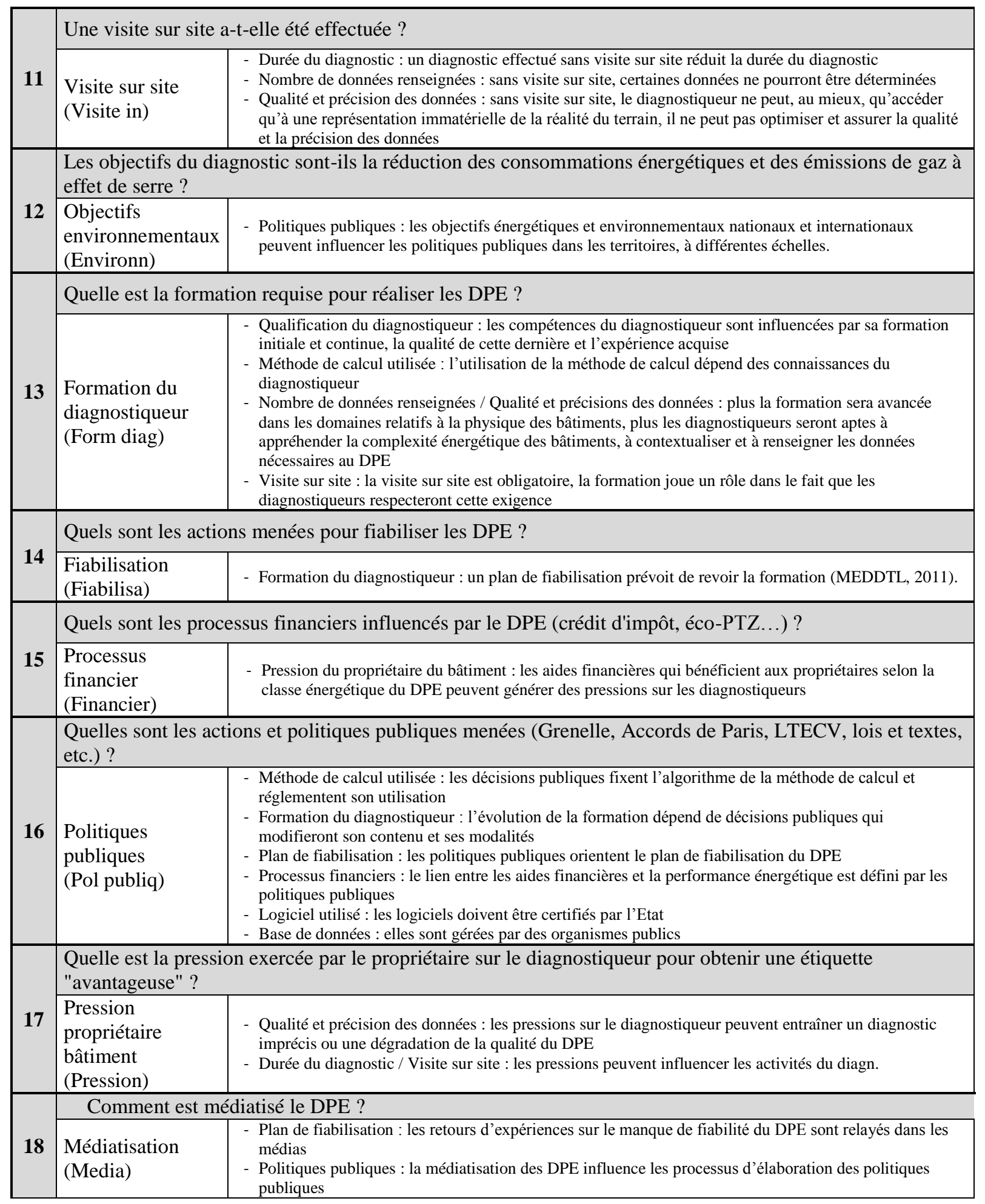

Table 1. Liste des variables associées aux sources d'erreurs et leurs influences directes 


\section{APPROCHE STRUCTURELLE DU SYSTÈME}

\section{Représentation matricielle directe}

Selon la méthode MICMAC, l'intensité des influences entre les variables est reportée dans une matrice dite des influences directes (Table 2). L'intensité prend la valeur 0 (nulle) si la variable de la ligne i n'a pas d'influence sur la variable de la colonne $\mathrm{j}$, et prend la valeur 1, 2 ou 3 (faible, moyenne, élevée) si la variable i a une influence directe faible, moyenne ou forte sur la variable $\mathrm{j}$.

\begin{tabular}{|c|c|c|c|c|c|c|c|c|c|c|c|c|c|c|c|c|c|c|}
\hline $\begin{array}{c}\text { Intensité de } \\
\text { l'influence de la } \\
\text { variable i (ligne) } \\
\text { sur la variable j } \\
\text { (colonne) } \\
\rightarrow\end{array}$ & 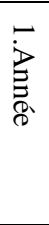 & 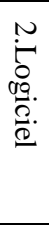 & 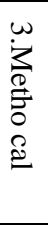 & 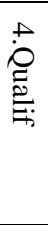 & 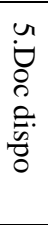 & 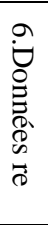 & 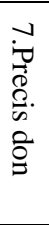 & 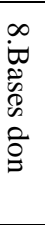 & 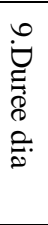 & 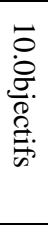 & 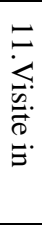 & 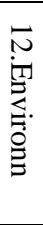 & 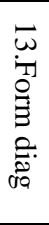 & 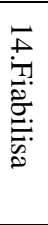 & 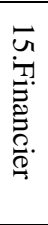 & $\begin{array}{l}\vec{a} \\
0 \\
0 \\
\overline{0} \\
0 \\
0 \\
0\end{array}$ & $\begin{array}{l}\vec{\nabla} \\
\overrightarrow{0} \\
0 \\
0 \\
0 \\
0 \\
0\end{array}$ & 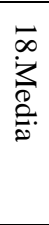 \\
\hline 1.Année & 0 & 0 & 3 & 0 & 0 & 0 & 1 & 0 & 2 & 0 & 1 & 0 & 0 & 0 & 0 & 0 & 0 & 0 \\
\hline 2.Logiciel & 0 & 0 & 0 & 0 & 0 & 2 & 0 & 0 & 1 & 0 & 0 & 0 & 0 & 0 & 0 & 0 & 0 & 0 \\
\hline 3.Metho cal & 0 & 2 & 0 & 0 & 0 & 3 & 2 & 0 & 2 & 0 & 0 & 0 & 0 & 0 & 0 & 0 & 0 & 0 \\
\hline 4.Qualif & 0 & 1 & 1 & 0 & 0 & 2 & 3 & 0 & 1 & 0 & 1 & 0 & 0 & 0 & 0 & 0 & 0 & 0 \\
\hline 5.Doc dispo & 0 & 0 & 0 & 0 & 0 & 1 & 2 & 0 & 0 & 0 & 1 & 0 & 0 & 0 & 0 & 0 & 0 & 0 \\
\hline 6.Données re & 0 & 0 & 0 & 0 & 0 & 0 & 0 & 1 & 0 & 0 & 0 & 0 & 0 & 0 & 0 & 0 & 0 & 0 \\
\hline 7.Precis don & 0 & 0 & 0 & 0 & 0 & 0 & 0 & 2 & 0 & 0 & 0 & 0 & 0 & 0 & 0 & 0 & 0 & 0 \\
\hline 8.Bases don & 0 & 0 & 0 & 0 & 0 & 0 & 0 & 0 & 0 & 0 & 0 & 2 & 0 & 0 & 0 & 1 & 0 & 0 \\
\hline 9.Duree dia & 0 & 0 & 0 & 0 & 0 & 3 & 3 & 0 & 0 & 0 & 0 & 0 & 0 & 0 & 0 & 0 & 0 & 0 \\
\hline 10.Objectifs & 0 & 0 & 0 & 0 & 0 & 0 & 1 & 0 & 1 & 0 & 0 & 1 & 0 & 0 & 0 & 0 & 0 & 0 \\
\hline 11.Visite in & 0 & 0 & 0 & 0 & 0 & 2 & 2 & 0 & 3 & 0 & 0 & 0 & 0 & 0 & 0 & 0 & 0 & 0 \\
\hline 12.Environn & 0 & 0 & 0 & 0 & 0 & 0 & 0 & 0 & 0 & 0 & 0 & 0 & 0 & 0 & 0 & 3 & 0 & 0 \\
\hline 13.Form diag & 0 & 0 & 2 & 3 & 0 & 1 & 1 & 0 & 0 & 0 & 1 & 0 & 0 & 0 & 0 & 0 & 0 & 0 \\
\hline 14.Fiabilisa & 0 & 0 & 0 & 0 & 0 & 0 & 0 & 0 & 0 & 0 & 0 & 0 & 2 & 0 & 0 & 0 & 0 & 0 \\
\hline 15.Financier & 0 & 0 & 0 & 0 & 0 & 0 & 0 & 0 & 0 & 0 & 0 & 0 & 0 & 0 & 0 & 0 & 3 & 0 \\
\hline 16.Pol publiq & 0 & 1 & 2 & 0 & 0 & 0 & 0 & 1 & 0 & 0 & 0 & 0 & 2 & 2 & 2 & 0 & 0 & 0 \\
\hline 17.Pression & 0 & 0 & 0 & 0 & 0 & 0 & 2 & 0 & 1 & 0 & 1 & 0 & 0 & 0 & 0 & 0 & 0 & 0 \\
\hline 18.Media & 0 & 0 & 0 & 0 & 0 & 0 & 0 & 0 & 0 & 0 & 0 & 0 & 0 & 2 & 0 & 2 & 0 & 0 \\
\hline
\end{tabular}

Table 2. Matrice des influences directes

Ainsi, d'après les retours d'expériences et l'étude bibliographique, il est considéré que la variable «13. Formation du diagnostiqueur» a une influence forte sur la variable «4. Qualification du diagnostiqueur », les compétences du diagnostiqueur étant très fortement influencées par sa formation, la qualité de cette dernière et les connaissances acquises. L'intensité de l'influence directe est notée 3 (Table 2).

Cette pondération des relations introduit une certaine dynamique dans l'analyse, et permet de tester la sensibilité des résultats en fonction de l'intensité des relations considérées.

Le remplissage de la matrice organise une procédure d'interrogation systématique qui alimente la réflexion sur la structure et le comportement du système.

Toutefois, cette matrice des influences directes reste un modèle qui ne permet pas de réduire la complexité du système au point de détecter les variables clés qu'il faudrait considérer en priorité. 


\section{Plan motricités-dépendances indirectes}

Selon la méthode MICMAC, l'étape suivante de l'analyse structurelle consiste à exploiter les propriétés des matrices par élévation en puissance de la matrice afin de considérer les influences indirectes entre les variables (Godet, 1991).

Cette matrice est donc élevée à la puissance 5 (prise en compte des influences indirectes correspondant à des chemins de longueur 5). La matrice obtenue fournit alors un classement des influences indirectes des variables qui permet d'établir le plan des motricités-dépendances indirectes ci-dessous (Figure 1).

Les résultats ainsi obtenus (matrices, classements, plans des influences/dépendances directes et indirectes) offre une vision systémique du système qui permet d'interroger la structure et l'évolution du système DPE représenté avec les 18 variables en interaction.

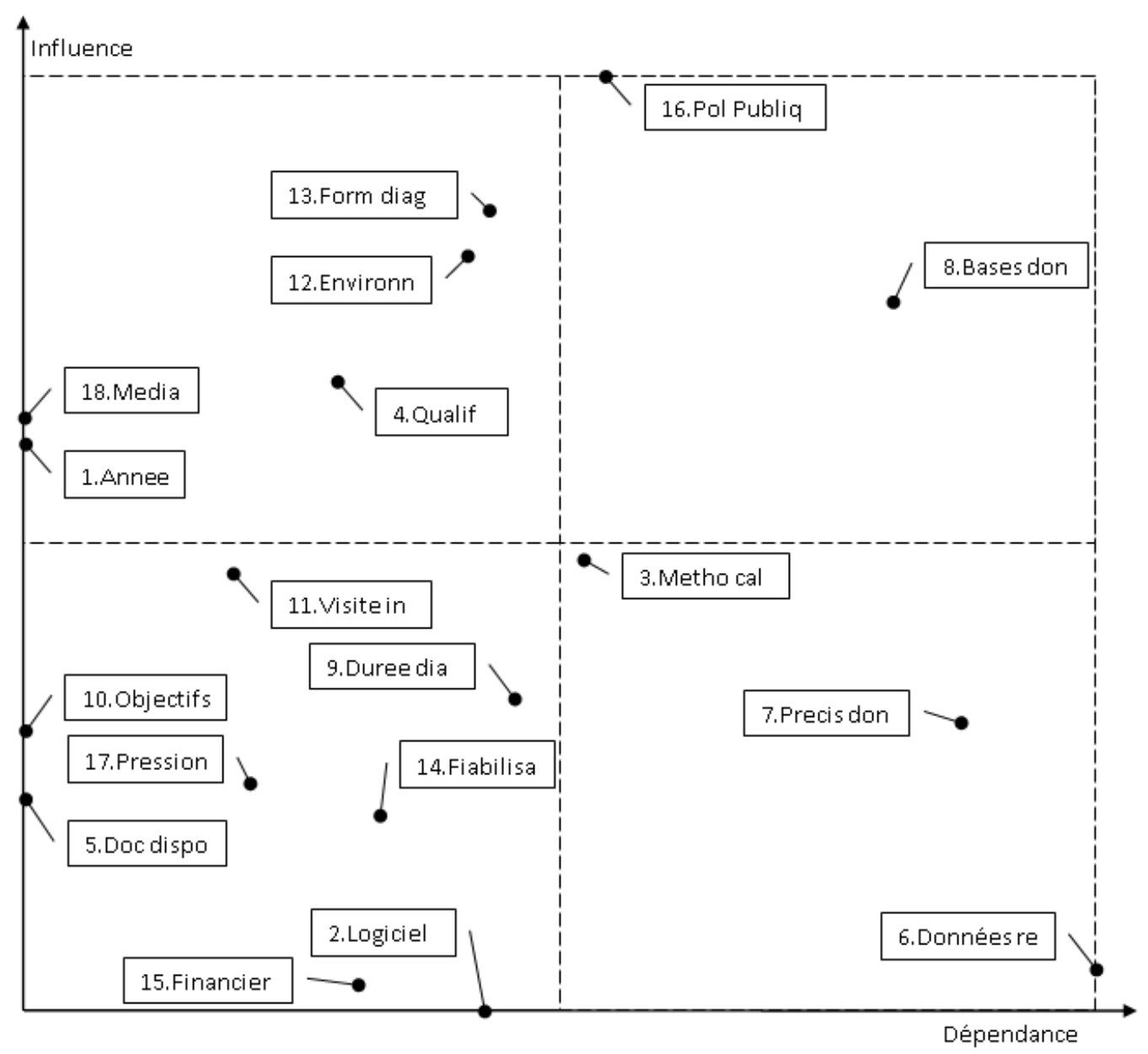

Figure 1. Plan des motricités-dépendances indirectes

Cette représentation du système (Figure 1) réduit la complexité du tissu relationnel entre les 18 variables du système. Le degré d'implication des variables dans le système peut être apprécié au travers des indicateurs de motricité et de dépendance. La motricité d'une variable mesure son influence sur le système et permet de l'identifier comme un facteur important d'évolution du système. La dépendance d'une variable rend compte de la façon dont celle-ci réagit aux changements d'autres variables du système.

Les classements et les plans motricités-dépendances directes et indirectes fournissent des représentations originales du système qui, par les questions qu'elles soulèvent, peuvent aider à mieux 
comprendre la dynamique de l'ensemble. La lecture attentive des plans est l'occasion de multiples commentaires et réflexions.

Ainsi, par exemple, la distribution des variables dans le plan caractérise une instabilité du système puisque deux variables «16. Politiques publiques » et « 8 . Bases de données » sont très motrices mais aussi très dépendantes. Il s'agit de variables dites relais qui concentrent les menaces et les opportunités du système, avec des influences fortes sur la «13. Formation des diagnostiqueurs », la «14. Fiabilisation du DPE » et les «15. Processus financier ».

La variable «16. Politiques publiques» est influencée par la variable «12. Objectifs environnementaux » mais aussi «18. Médiatisation». Cette variable «16. Politiques publiques» influence indirectement le «6. Nombre de données renseignées » dans le DPE mais aussi l'exigence de «7. Qualité et précision des données » et donc les «8. Bases de données ». Le plan motricitésdépendances fait apparaître le rôle essentiel dans l'évolution du système des variables «16. Politiques publiques » et des différentes variables relatives aux données («8. Bases de données », «7. Précision et qualité des données », «6. Nombre de données renseignées »).

La variable «13. Formation des diagnostiqueurs » joue également un rôle important dans le système puisqu'elle est très motrice. Elle constitue un levier pour agir sur le système. La qualité du DPE est fortement influencée par le diagnostiqueur et donc par la qualité de sa formation. De solides connaissances en construction, physique et modélisation énergétique du bâtiment sont essentielles pour appréhender les problématiques complexes liées à l'évaluation de la performance énergétique et à l'identification des scénarios de réhabilitation les plus pertinents répondant aux exigences d'un développement durable.

Enfin, la variable «1. Année de construction » du bâtiment est motrice dans le système. Elle constitue en quelque sorte une entrée du système, une hypothèse forte. Cette variable détermine le choix de la méthode de calcul utilisée et influence la durée du diagnostic. Les retours d'expériences ont montré que les erreurs pouvaient être importantes lorsque l'année de construction n'était pas connue ou lorsque la construction était ancienne.

Les variables relatives aux données «7. Qualité et précision des données » et « 6 . Nombre de données renseignées » sont fortement dépendantes. Ce sont des variables très sensibles et extrêmement hétérogènes d'un diagnostic à l'autre. Elles sont très fortement impactées par d'autres variables, notamment celles relatives au diagnostiqueur et à la méthode de calcul.

La variable «5. Documents à disposition des diagnostiqueurs », peu motrice et sans dépendance, semble exclue du système. Ce résultat parait contre-intuitif. Il en est de même pour les variables ayant une faible motricité et une faible dépendance telles que « Pression du propriétaire du bâtiment », «15. Processus financier » ou «2. Logiciel utilisé ». S'agit-il de variables définies à partir d'une fausse représentation du système considéré à l'échelle de sa globalité ? Une analyse plus approfondie devrait aider à comprendre le rôle de ces variables.

Cette mise en œuvre de l'analyse structurelle pour la recherche des variables clés du système permet d'explorer la complexité structurelle du système. Même si elle paraît parfois conforter des résultats connus, elle révèle certains aspects contre-intuitifs du système qui questionnent, à l'échelle globale du DPE, le rôle du «15. Processus financier», du «2. Logiciel utilisé » ou des «5. Documents mis à disposition des diagnostiqueurs ».

\section{CONCLUSION}

Depuis plusieurs années, les retours d'expériences et diverses études interrogent la fiabilité et la valeur informative des diagnostics de performance énergétique, et donc la pertinence des représentations et des mesures de réhabilitation qu'ils proposent. Même si le DPE n'a pas vocation de prescrire des solutions (MTES. 2018b), ils génèrent des données, des activités, des avis, des recommandations et diverses représentations qui influencent le marché immobilier, orientent les décisions et les actions de réhabilitation énergétique. 
Le caractère multifactoriel des sources d'erreurs met en exergue la problématique complexe du diagnostic de performance énergétique pour les bâtiments. Les retours d'expériences confirment que cette complexité ne peut être que partiellement appréhendée par les approches simplificatrices du DPE. La complexité du DPE est difficile à maîtriser sans les connaissances et les compétences permettant de concevoir et d'ajuster un modèle énergétique adapté à chaque bâtiment.

Dans ce contexte, complètant l'approche analytique, l'approche systémique, mise en œuvre par une analyse structurelle, fait apparaitre les principaux facteurs d'évolution du système DPE.

Le résultat sans doute le plus important de cette étude est d'avoir mis en évidence l'apport de la vision systémique qui offre une appréhension nouvelle des sources d'erreurs et des déterminants de l'évolution du DPE. Le recensement de 18 variables identifiées à partir des retours d'expériences permet en effet de se doter de représentations originales du système DPE et d'en réduire la complexité avec la méthode MICMAC.

Les résultats obtenus montrent que c'est autour des variables relais et des variables motrices que peuvent s'élaborer de nouvelles options stratégiques pour le DPE. Les variables relais sont les variables «Politiques publiques » et «Bases de données». Les variables motrices sont la «Formation des diagnostiqueurs », les «Objectifs environnementaux du DPE », la «Qualification du diagnostiqueur », la « Médiatisation » et l'« Année de construction».

Ces résultats permettent également de considérer des aspects contre-intuitifs du système lorsque les représentations font apparaître que des variables telles que "Documents à disposition des diagnostiqueurs », «Pression du propriétaire du bâtiment », «Processus financier » et « Logiciel utilisé » sont peu influentes et peu dépendantes.

Cette étude montre que des pistes d'amélioration du DPE peuvent être envisagées en considérant les problématiques de politiques publiques, de production et d'utilisation des données relatives à la performance énergétique des bâtiments. Si le caractère multifactoriel des sources d'erreurs peut être la cause d'une forme de renoncement et d'inaction face aux difficultés rencontrées, en raison parfois de l'incapacité à appréhender la complexité des problèmes, il apparaît nécessaire de recourir à une pensée complexe (Morin, 2007. Perriault et al., 2011). L'approche systémique stimule la réflexion pour explorer de nouvelles réponses aux défis d'un développement durable en considérant les enjeux d'une vigilance épistémique (Fleurance, 2018) et le rôle des données.

Enfin, cette étude initiée avec l'analyse structurelle pourrait être discutée, consolidée et approfondie en mobilisant un ou des groupes de réflexion prospective (Godet, 1991) autour de cette problématique complexe du DPE, possible levier d'une transition énergétique et environnementale du secteur du bâtiment.

\section{REFERENCES}

ADEME. (2018). Bienvenue sur l'espace DPE. Diagnostic de Performance Energétique. http://www.observatoire-dpe.fr/

Amecke, H. (2012). The impact of energy performance certicates: A survey of German home owners. Energy Policy, 46, pp. 4-14.

Bjorn, H., Daghbashyan, Z., \& Chaudhary, P. (2016). On the quality and impact of residential energy performance certificates. Energy and Buildings, 133, pp. 711-723.

Cantin, R. Colomb, V. et Jalenques-Vigouroux, B. (2013). Approche interdiscipliniare de la requalification énergétique du logement social. La place des chiffres dans une étude mêlant sciences de l'ingénieur et sciences de l'information et de a communication. $8^{\text {ème }}$ congrès international Réseau International de recherche sur les Organisations et le Développement Durable, RIODD 2013. Lille, 1821 juin 2013, 26p.

Donnadieu,G. Durand, D. Neel, D. Nunez, E. \& Saint-Paul, L. (2003). L'Approche systémique : de quoi s'agit-il ? AFSCET, Paris. 
DINAMIC. (2015). La valeure verte des logements d'après les bases notariales BIEN et PERVAL. Durand, D. (1994). La systémique. PUF, Paris.

Fleurance, P. (2018). Les données sont partout...! Questions sur le "datafication". Un exercice de vigilance épistémique et citoyen? Site internet Réseau Intelligence de la Complexité http://www.mcxapc.org/

Fuerst, F. Mcallister, P. Nanda, A. \& Wyatt, P. (2013). An investigation of the effect of EPC ratings on house prices in the UK. Department of Energy \& Climate Change.

Godet, M. (1991). De l'anticipation à l'action. Manuel de prospective stratégique. Dunod, Paris.

Jenkins, D., Simpson, S., \& Peacock, A. (2017). Investigating the consistency and quality of EPC ratings and assessments. Energy, 138, pp. 480-489.

Le Moigne, J.L. (1994). Théorie du système général. Vendôme, PUF. Paris.

Le Moigne, J.L. (1995). La modélisation des systèmes complexes. Afcet Systèmes. Dunod. Paris.

MEDDTL. 2011. Ministère de l'écologie, du développement durable, des transports et du logement.

Plan d'action du Ministère pour la fiabilisation du diagnostic de performance énergétique. Dossier de presse. 6 mesures pour améliorer le Diagnostic de Performance Energétique (DPE).

https://www.ecologique-solidaire.gouv.fr/diagnostics-techniques-immobiliers [fichier pdf 24/9/2018].

Ministère du logement. (2009). Diagnostic de performance énergétique - Guide des recommandation

Morin, E. (1977). La méthode, la nature de la nature. Seuil, Paris.

Morin, E. (2007). Introduction à la pensée complexe. Points, Paris.

MTES. (2018a). Diagnostics techniques immobiliers. Ministère de la Transition écologique et solidaire https://www.ecologique-solidaire.gouv.fr/diagnostics-techniques-immobiliers

MTES. (2018b). Diagnostic de performance énergétique - DPE. Ministère de la Transition écologique et solidaire. https://www.ecologique-solidaire.gouv.fr/diagnostic-performance-energetiquedpe.

Perriault. J., Proutheau. S., Kleinpeter. E., Pena-Vega. A. et Le Moigne J-L. (2011). L'exercice de la pensée complexe permet l'intelligence des systèmes complexes. Entretien. Hermès, La Revue 2011/2 $\left(n^{\circ} 60\right)$, p. 157-163.

Rosnay, de J. (1975). Le macroscope, vers une vision globale. Seuil, Paris.

Stone. A., Shipworth, D., Biddulph. P., \& Oreszczyn, T. (2014) Key factors determining the energy rating of existing English houses, Building Research \& Information, 42:6, 725-738.

Thammasitboon, S. Thammasitboon, S. \& Singhal, G. (2013). Diagnosing diagnostic error. Current problems in pediatric and adolescent health care, 43. pp 227-231.

Tronchin, L. \& Fabbri, K. (2012). Energy performance certificate of building and confidence interval in assessment : An Italian case study. Energy policy, 48, pp. 176-184.

Tudela, P., Carreres, A. \& Ballester, M. (2017). Diagnostic errors in emergency departments. 149, pp. 170-175.

UFC. (2017). Union Fédérale des Consommateurs - Que Choisir. Diagnostics de performance énergétique. Stop à la loterie! Site internet https://www.quechoisir.org/ . 